\title{
Branched Chain Amino Acid Supplementation to a Hypocaloric Diet Does Not Affect Resting Metabolic Rate but Increases Postprandial Fat Oxidation Response in Overweight and Obese Adults after Weight Loss Intervention
}

\author{
Delicia Shu Qin Ooi ${ }^{1,2}{ }^{\circledR}$, Jennifer Qiu Rong Ling ${ }^{1,2}$, Fang Yi Ong ${ }^{1,2}$, E Shyong Tai ${ }^{3}$, \\ Christiani Jeyakumar Henry ${ }^{4}$, Melvin Khee Shing Leow ${ }^{5,6,7} \mathbb{D}^{\circ}$, Eric Yin Hao Khoo ${ }^{3}$, Chuen Seng Tan ${ }^{8}$, \\ Mary Foong Fong Chong ${ }^{4,5,8}$, Chin Meng Khoo ${ }^{3, \dagger}$ and Yung Seng Lee ${ }^{1,2,5, *, \dagger}$
}

Citation: Ooi, D.S.Q.; Ling, J.Q.R.; Ong, F.Y.; Tai, E.S.; Henry, C.J.; Leow, M.K.S.; Khoo, E.Y.H.; Tan, C.S.; Chong, M.F.F.; Khoo, C.M.; et al. Branched Chain Amino Acid Supplementation to a Hypocaloric Diet Does Not Affect Resting Metabolic Rate but Increases Postprandial Fat Oxidation Response in Overweight and Obese Adults after Weight Loss Intervention. Nutrients 2021, 13, 4245. https:// doi.org/10.3390/nu13124245

Academic Editor: Roberto Iacone

Received: 26 September 2021 Accepted: 24 November 2021 Published: 26 November 2021

Publisher's Note: MDPI stays neutral with regard to jurisdictional claims in published maps and institutional affiliations.

Copyright: (c) 2021 by the authors. Licensee MDPI, Basel, Switzerland. This article is an open access article distributed under the terms and conditions of the Creative Commons Attribution (CC BY) license (https:// creativecommons.org/licenses/by/ $4.0 /)$.
1 Department of Paediatrics, Yong Loo Lin School of Medicine, National University of Singapore, Singapore 117549, Singapore; paeosqd@nus.edu.sg (D.S.Q.O.); qrongling@gmail.com (J.Q.R.L.); fangyi_o@yahoo.com (F.Y.O.)

2 Khoo Teck Puat-National University Children's Medical Institute, National University Health System, Singapore 119074, Singapore

3 Department of Medicine, Yong Loo Lin School of Medicine, National University of Singapore, Singapore 117549, Singapore; mdctes@nus.edu.sg (E.S.T.); mdckyhe@nus.edu.sg (E.Y.H.K.); mdckcm@nus.edu.sg (C.M.K.)

4 Clinical Nutrition Research Center, Agency for Science, Technology and Research, Singapore 117599, Singapore; bchchjh@nus.edu.sg (C.J.H.); mary_chong@nus.edu.sg (M.F.F.C.)

5 Singapore Institute for Clinical Sciences, Agency for Science, Technology and Research, Singapore 117609, Singapore; melvin_leow@sics.a-star.edu.sg

6 Department of Endocrinology, Division of Medicine, Tan Tock Seng Hospital, Singapore 308433, Singapore

7 Lee Kong Chian School of Medicine, Nanyang Technological University, Singapore 639798, Singapore

8 Saw Swee Hock School of Public Health, National University of Singapore, Singapore 117549, Singapore; ephtcs@nus.edu.sg

* Correspondence: paeleeys@nus.edu.sg; Tel.: +65-6772-4111

$\dagger$ Joint last authors.

Abstract: Background: Branched chain amino acids (BCAA) supplementation is reported to aid in lean mass preservation, which may in turn minimize the reduction in resting metabolic rate (RMR) during weight loss. Our study aimed to examine the effect of BCAA supplementation to a hypocaloric diet on RMR and substrate utilization during a weight loss intervention. Methods: A total of 111 Chinese subjects comprising 55 males and 56 females aged 21 to 45 years old with BMI between 25 and $36 \mathrm{~kg} / \mathrm{m}^{2}$ were randomized into three hypocaloric diet groups: (1) standardprotein (14\%) with placebo (CT), (2) standard-protein with BCAA, and (3) high-protein (27\%) with placebo. Indirect calorimetry was used to measure RMR, carbohydrate, and fat oxidation before and after 16 weeks of dietary intervention. Results: RMR was reduced from $1600 \pm 270 \mathrm{kcal} /$ day to $1500 \pm 264 \mathrm{kcal} /$ day $(p<0.0005)$ after weight loss, but no significant differences in the change of RMR, respiratory quotient, and percentage of fat and carbohydrate oxidation were observed among the three diet groups. Subjects with BCAA supplementation had an increased postprandial fat $(p=0.021)$ and decreased postprandial carbohydrate $(p=0.044)$ oxidation responses compared to the CT group after dietary intervention. Conclusions: BCAA-supplemented standard-protein diet did not significantly attenuate reduction of RMR compared to standard-protein and high-protein diets. However, the postprandial fat oxidation response increased after BCAA-supplemented weight loss intervention.

Keywords: branched chain amino acids (BCAA); resting metabolic rate (RMR); diet-induced thermogenesis (DIT); respiratory quotient (RQ); carbohydrate oxidation; lipid oxidation; hypocaloric diet; high-protein; weight loss; obese and overweight adults 


\section{Introduction}

Resting metabolic rate (RMR) and substrate utilization are crucial in maintaining energy balance, which underlies the regulation of body weight [1].

RMR, also termed as resting energy expenditure (REE), is defined as the energy required by the body when in resting condition without any physical activity [2]. RMR accounts for most of the body's energy needs and expenditure [3], and differences in body composition influence an individual's RMR [4]. Variability in the fat mass and lean mass (fat free mass) are associated with variability in RMR [5,6]. In particular, an increase in lean mass is correlated with a higher RMR [5,7].

It is well-established that energy expenditure, including RMR decreases after weight loss $[8,9]$. This reduction in RMR is known as metabolic adaptation (also termed adaptive thermogenesis) $[10,11]$. During weight loss, the energy expenditure exceeds energy intake resulting in a negative energy balance. Metabolic adaptation will be directed towards energy sparing, which is the lowering of RMR to compensate for the negative energy balance [12,13]. A low RMR is regarded as a risk factor for weight gain [14,15], and metabolic adaptation after weight loss is also a predictor of weight regain [16]. Hence, minimizing the loss of RMR during or after weight loss might reduce subsequent regain of weight.

Substrate utilization is indicated by the respiratory quotient (RQ). A high fasting $\mathrm{RQ}$ corresponds to carbohydrate oxidation, while a low fasting RQ corresponds to fat oxidation [17]. Individuals with a high fasting $R Q$ had greater gains in body weight and fat mass compared to a low/moderate fasting RQ among free-living healthy young adults [18]. $\mathrm{RQ}$ is positively correlated with total body fat mass, trunk fat mass, and visceral adipose tissues [19]. In addition, a lower postprandial RQ corresponding to higher fat oxidation is also associated with significant reductions in fat mass and body weight [20].

Protein is an essential macronutrient for building muscle mass (lean mass) [21], and a higher protein intake is associated with higher lean mass and muscle strength [22]. Highprotein hypocaloric diets have been shown to preserve lean mass during weight loss [23-26], and a protein-enriched diet was found to mitigate the decrease in lean mass and RMR in obese subjects who underwent sleeve gastrectomy [27]. Branched chain amino acid (BCAA: valine, leucine, and isoleucine), which forms one-third of total muscle protein, can stimulate skeletal muscle regeneration [28] and suppress post-exercise muscle degradation [29,30]. In individuals who undergo resistance training, a BCAA-supplemented hypocaloric diet is able to maintain lean mass and preserve skeletal muscle performance while losing fat mass [31]. BCAA supplementation to a moderate protein hypocaloric diet was found to cause the highest body weight loss and decrease in percent body fat, as well as a significant reduction in abdominal visceral adipose tissue in a group of competitive wrestlers [32]. However, some studies did not observe an effect of BCAA supplementation on body weight, lean mass, and fat mass in overweight and obese adults after a hypocaloric diet-induced weight loss [33,34].

Preliminary studies showed that BCAA supplementation increased RMR in human adults $[35,36]$. The knockdown of branched-chain aminotransferase (BCAT) gene that encodes the enzyme involved in BCAA catabolism resulted in increased plasma BCAAs, decreased adiposity and body weight, and increased energy expenditure in mice [37]. In addition, dietary BCAA restriction has been found to alter substrate utilization in Zucker fatty rats [38], and BCAA supplementation was shown to enhance lipid oxidation during exercise [39]. However, the effect of BCAA supplementation on RMR and substrate utilization during hypocaloric diet-induced weight loss intervention in overweight and obese adults has not been investigated.

This study compared the change in RMR and substrate utilization between diet groups with different protein content supplemented with or without BCAA during weight loss. We hypothesize that a BCAA-supplemented standard-protein hypocaloric diet would minimize the decrease in RMR and alter substrate utilization during a weight loss intervention. 


\section{Materials and Methods}

\subsection{Study Participants}

The study participants were part of a randomized, controlled trial registered at clincialtrials.gov (accessed on 10 November 2021) as NCT02277275. The trial aimed to examine the effect of BCAA supplementation on changes in primary outcomes including lean and fat mass, and changes in secondary outcomes including insulin sensitivity, RMR, and diet-induced thermogenesis (DIT). We have previously reported the effect of BCAA supplementation on lean mass preservation and insulin sensitivity of the participants in the trial [34]. In this study, we included 111 subjects who had completed indirect calorimetry after 16 weeks of weight loss intervention. On the basis of mean change of RMR ( $-412 \pm 67 \mathrm{kcal} /$ day) after 8 weeks of calorically restricted diet supplemented with BCAA [31], we found our sample of 111 subjects to be more than adequately powered ( $>90 \%$ ) at $\alpha=0.05$ to detect differences in RMR between hypocaloric diet groups supplemented with and without BCAA [40]. Out of these 111 subjects, 109 subjects completed the 24 week intervention study (Figure S1). The subjects were Chinese men and women, aged between 21 and 45 years old. They were either overweight or obese, with BMI ranging between 25 and $36 \mathrm{~kg} / \mathrm{m}^{2}$, and with percent body fat $\geq 25 \%$. All of them had no existing medical conditions (e.g., diabetes mellitus; hypertension; and kidney, liver, and thyroid problems). All procedures were in accordance with the Declaration of Helsinki, as revised in 2013. Written informed consent was obtained from all subjects, and this study was approved by the Domain Specific Review Board of National Healthcare Group, Singapore.

\subsection{Study Design}

The study design for this trial has been described previously [34]. The study participants were block randomized by gender and BMI groups (25-29 kg/m² versus $30-36 \mathrm{~kg} / \mathrm{m}^{2}$ ) into three hypocaloric diet groups: (1) standard-protein diet with placebo supplementation (control, CT), (2) standard-protein diet with BCAA supplementation (BCAA), and (3) highprotein diet with placebo supplementation (HP). Since amino acids are the building blocks of proteins, we included the HP diet group to examine if the supplementation of standardprotein diet with BCAA could attain the same outcomes as that of a high-protein diet. The BCAA diet is the experimental diet intervention, while the CT and HP diets are placebo diets that served as controls for assessing the effect of BCAA supplementation on the study outcomes. The three diets were designed to induce an energy deficit of $500 \mathrm{kcal}$ per day. All subjects underwent 16 weeks of dietary weight loss intervention with prescribed meals (Charoen Pokphand Intertrade (PTE) Ltd., Singapore, JR Foods (PTE) Ltd., Singapore) and daily supplements (BCAA or placebo), which were provided according to body weight (BCAA: $0.1 \mathrm{~g} \cdot \mathrm{kg}^{-1}$ body weight $\cdot \mathrm{d}^{-1}$ divided into three doses per day with main meals, maximum $11.05 \mathrm{~g} /$ day). The proposed dose of BCAA at $0.1 \mathrm{~g} \cdot \mathrm{kg}^{-1}$ body weight $\cdot \mathrm{d}^{-1}$ was based on the typical dose used in other studies $[30,41,42]$, and the maximum amount given was below the upper tolerable limit of BCAA [43]. Weekly food delivery for 6 days' worth of food was provided throughout the 16 weeks, and subjects had one 'free day' each week of their own food. The placebo and BCAA supplements were provided throughout the study. The BCAA (AST BCAA 4500, Golden, CO, USA) had $325 \mathrm{mg}$ L-leucine, $162.5 \mathrm{mg}$ L-isoleucine, and $162.5 \mathrm{mg}$ L-valine per tablet with no other added vitamins, minerals, and nutrients. Placebo was manufactured (Beacons Pharmaceuticals Pte Ltd., Singapore) to resemble the BCAA in appearance but consisted of corn-starch and magnesium stearate.

To document diet compliance, subjects checked off food checklists sent with their weekly food delivery and recorded extra foods and beverages consumed. The dietitian provided diet counselling sessions at weeks $0,4,8,12$, and 16 , and also called subjects fortnightly to assess and encourage compliance.

The subjects included in this study attended 2 study visits: baseline (week 0 ) and after controlled dietary intervention (week 16). At each study visit (week 0 and week 16), the subjects consumed a $600 \mathrm{kcal}$ test meal representative of their study diet within $20 \mathrm{~min}$. The test meal was in the form of a packaged meal and a drink. The standard-protein test 
meal consisted of $14 \%$ protein, $56 \%$ carbohydrate, and $30 \%$ fat, and the amount of BCAA additionally provided to the BCCA group was approximately half the dose of a day's allocated intake. The high-protein test meal consisted of $27 \%$ protein, $43 \%$ carbohydrate, and $30 \%$ fat.

\subsection{Anthropometric and Biochemical Measurements}

Anthropometric measurements including height, weight, waist circumference, and blood pressure were recorded [34]. Height and weight were taken using a Seca 763 Digital scale. Blood pressure was taken after subjects rested for at least $20 \mathrm{~min}$ using an Omron Digital Automatic Blood Pressure Monitor (Model HEM-907). Body composition (percent body fat, fat mass, and lean mass) was measured using a Dual-energy X-ray Absorptiometry System (DXA) (Hologic, Santa Clara, CA, USA, model: ASY-05119).

Fasting blood samples were obtained before consumption of test meal during the 2 study visits: week 0 and week 16 for the measurement of plasma glucose (photometric assay, hexokinase method), insulin (immunoenzymatic assay), and lipids (enzymatic colorimetric assay) levels [34]. Homeostatic model assessment of insulin resistance (HOMA-IR) was calculated as previously described [44]. Blood samples were also collected at $180 \mathrm{~min}$ after test meal for the measurement of plasma glucose and insulin levels.

Subjects were asked to maintain their baseline physical activity level throughout the study. All subjects were provided with an accelerometer (ActiGraph, Pensacola, FL, USA) at week 0 and 8 visits, and the accelerometer was worn for a week at both visits. The data were used to calculate metabolic equivalent of task (MET) and percentage of moderate to vigorous-intensity physical activity (\%MVPA). There were no significant differences for MET or \%MVPA among subjects in the different diet groups [34].

\subsection{Indirect Calorimetry}

RMR and substrate utilization were assessed using an open-circuit indirect calorimetry system that measures the amount of oxygen consumption (VO2) and amount of carbon dioxide production (VCO2). After an 8 h overnight fast, subjects laid supine on a bed in a thermoneutral environment with a clear plastic hood over their head and shoulders, and $\mathrm{VO} 2$ and $\mathrm{VCO} 2$ were recorded for $30 \mathrm{~min}$ (Quark CPET, COSMED, Rome, Italy). The first $10 \mathrm{~min}$ of data were discarded to ensure all subjects had reached equilibrium, and the remaining $20 \mathrm{~min}$ of data were averaged to calculate resting metabolic rate (RMR), respiratory quotient $(R Q)$, percentage of fat oxidation, and percentage of carbohydrate oxidation [45,46]. RMR is calculated using the Weir formula [47], and RQ is calculated as $\mathrm{VCO} 2 / \mathrm{VO} 2$. $\mathrm{RQ}$ provides an estimate for transition between percentage of fat $(\mathrm{RQ}=0.7)$ and carbohydrate oxidation $(R Q=1.0)$ [48]. After the measurement at fasting state, each subject consumed a $600 \mathrm{kcal}$ test meal representative of their study diet within $20 \mathrm{~min}$, and $\mathrm{VO} 2$ and VCO2 were recorded continuously for a subsequent $3 \mathrm{~h}$.

\subsection{Statistical Analysis}

All analyses were performed using SPSS 26.0 with level of statistical significance set at two-sided $p<0.05$. Descriptive statistics for numerical and categorical variables are presented as mean \pm standard deviation (mean $\pm \mathrm{SD}$ ) and $\mathrm{N}$, respectively. Differences in clinical characteristics among the three diet groups were analyzed by one-way ANOVA with Bonferroni correction for continuous parameters and chi-squared for categorical parameters. Paired $t$-test was used to analyze differences in clinical characteristics before (week 0) and after (week 16) dietary intervention. Spearman's correlation was used to correlate change in RMR and substrate utilization (week 16 vs. week 0 ) with change in metabolic parameters (week 16 vs. week 0). Repeated measures ANOVA (within subject group) was used to compare postprandial $R Q$, fat oxidation, carbohydrate oxidation, and DIT responses after test meal between week 0 and week 16 , and between subjects in the three diet groups. 


\section{Results}

\subsection{Clinical Characteristics of Study Participants}

There were no significant differences in the baseline (week 0 ) characteristics for gender, age, weight, BMI, waist circumference, percent body fat, fat and lean mass, blood pressure, $\mathrm{RMR}$, fat oxidation, carbohydrate oxidation, $\mathrm{RQ}$, lipid profile and parameters of glucose homeostasis among the three diet groups (Table S1).

After 16 weeks of weight loss intervention, the study participants showed significant reductions in weight, BMI, waist circumference, percent body fat, total body fat mass, total body lean mass, systolic blood pressure, diastolic blood pressure, RMR, total cholesterol, triglycerides, HDL cholesterol, LDL cholesterol, fasting glucose, fasting insulin, and HOMA-IR (Table S2).

However, there were no significant differences in the absolute change in RMR, RQ, and metabolic parameters among the three diet groups after 16 weeks of weight loss intervention (Table 1).

Table 1. Change in clinical parameters among study participants in the three diet groups after 16 weeks of weight loss intervention.

\begin{tabular}{|c|c|c|c|c|}
\hline Parameters & CT $(n=37)$ & BCAA $(n=35)$ & $\mathrm{HP}(n=39)$ & $p$ \\
\hline Weight (kg) & $-6.62 \pm 3.44$ & $-6.39 \pm 3.99$ & $-6.35 \pm 2.37$ & 0.931 \\
\hline $\mathrm{BMI}\left(\mathrm{kg} / \mathrm{m}^{2}\right)$ & $-2.45 \pm 1.36$ & $-2.26 \pm 1.33$ & $-2.33 \pm 0.811$ & 0.780 \\
\hline Waist circumference $(\mathrm{cm})$ & $-6.32 \pm 3.69$ & $-7.43 \pm 4.55$ & $-7.18 \pm 3.12$ & 0.427 \\
\hline Body fat percentage (\%) & $-2.34 \pm 1.95$ & $-2.37 \pm 2.41$ & $-2.75 \pm 1.92$ & 0.631 \\
\hline Total body fat mass (kg) & $-4.23 \pm 2.24$ & $-4.09 \pm 2.88$ & $-4.34 \pm 1.91$ & 0.902 \\
\hline Total body lean mass (kg) & $-2.39 \pm 1.50$ & $-2.15 \pm 1.74$ & $-1.80 \pm 1.52$ & 0.272 \\
\hline RMR (kcal/day) & $-98.6 \pm 165$ & $-108 \pm 119$ & $-90.8 \pm 140$ & 0.874 \\
\hline Fat $(\%)$ & $-2.35 \pm 27.0$ & $4.06 \pm 23.4$ & $-0.95 \pm 32.6$ & 0.599 \\
\hline $\mathrm{CHO}(\%)$ & $2.35 \pm 26.9$ & $-4.09 \pm 23.4$ & $0.95 \pm 32.5$ & 0.595 \\
\hline $\mathrm{RQ}$ & $0.008 \pm 0.09$ & $-0.012 \pm 0.07$ & $0.006 \pm 0.11$ & 0.599 \\
\hline Total cholesterol (mmol/L) & $-0.32 \pm 0.60$ & $-0.25 \pm 0.51$ & $-0.36 \pm 0.48$ & 0.648 \\
\hline Triglycerides (mmol/L) & $-0.13 \pm 0.38$ & $-0.17 \pm 0.54$ & $-0.23 \pm 0.31$ & 0.581 \\
\hline HDL cholesterol (mmol/L) & $-0.08 \pm 0.13$ & $-0.07 \pm 0.14$ & $-0.05 \pm 0.12$ & 0.469 \\
\hline LDL cholesterol (mmol/L) & $-0.17 \pm 0.47$ & $-0.10 \pm 0.49$ & $-0.21 \pm 0.40$ & 0.585 \\
\hline Fasting glucose (mmol/L) & $-0.19 \pm 0.32$ & $-0.15 \pm 0.25$ & $-0.16 \pm 0.31$ & 0.874 \\
\hline Fasting insulin (mU/L) & $-3.54 \pm 4.21$ & $-3.60 \pm 4.96$ & $-2.96 \pm 3.77$ & 0.779 \\
\hline HOMA-IR & $-0.84 \pm 1.06$ & $-0.79 \pm 1.03$ & $-0.68 \pm 0.91$ & 0.768 \\
\hline Systolic blood pressure (mmHg) & $-3 \pm 8$ & $-4 \pm 9$ & $-3 \pm 12$ & 0.932 \\
\hline Diastolic blood pressure (mmHg) & $-3 \pm 9$ & $-6 \pm 6$ & $-2 \pm 11$ & 0.138 \\
\hline
\end{tabular}

Data presented as mean change \pm SD. One-way ANOVA with Bonferroni correction was used to compare differences in absolute change in clinical parameters (week 16 vs. week 0) among the 3 diet groups. Abbreviations: standard-protein with BCAA, BCAA; standard-protein with placebo, CT; carbohydrates, $\mathrm{CHO}$; high-density lipoprotein, HDL; high-protein with placebo, HP; homeostatic model assessment of insulin resistance, HOMA-IR; low-density lipoprotein, LDL; resting metabolic rate, RMR; respiratory quotient, RQ.

\subsection{Correlation between Change in RMR and Change in Metabolic Measures after 16 Weeks of Weight Loss Intervention (Week 16 vs. Week 0)}

The change in RMR was positively correlated with the change in weight, BMI, and total body fat mass (Figure 1). A decrease in RMR was correlated with a decrease in weight $(\mathrm{r}=0.225, p=0.017)$ (Figure 1A), BMI $(\mathrm{r}=0.206, p=0.030)$ (Figure 1B), and total body fat mass $(r=0.193, p=0.042)$ (Figure 1C). The change in RMR was not significantly correlated with the change in metabolic parameters within each diet group (data not shown). 

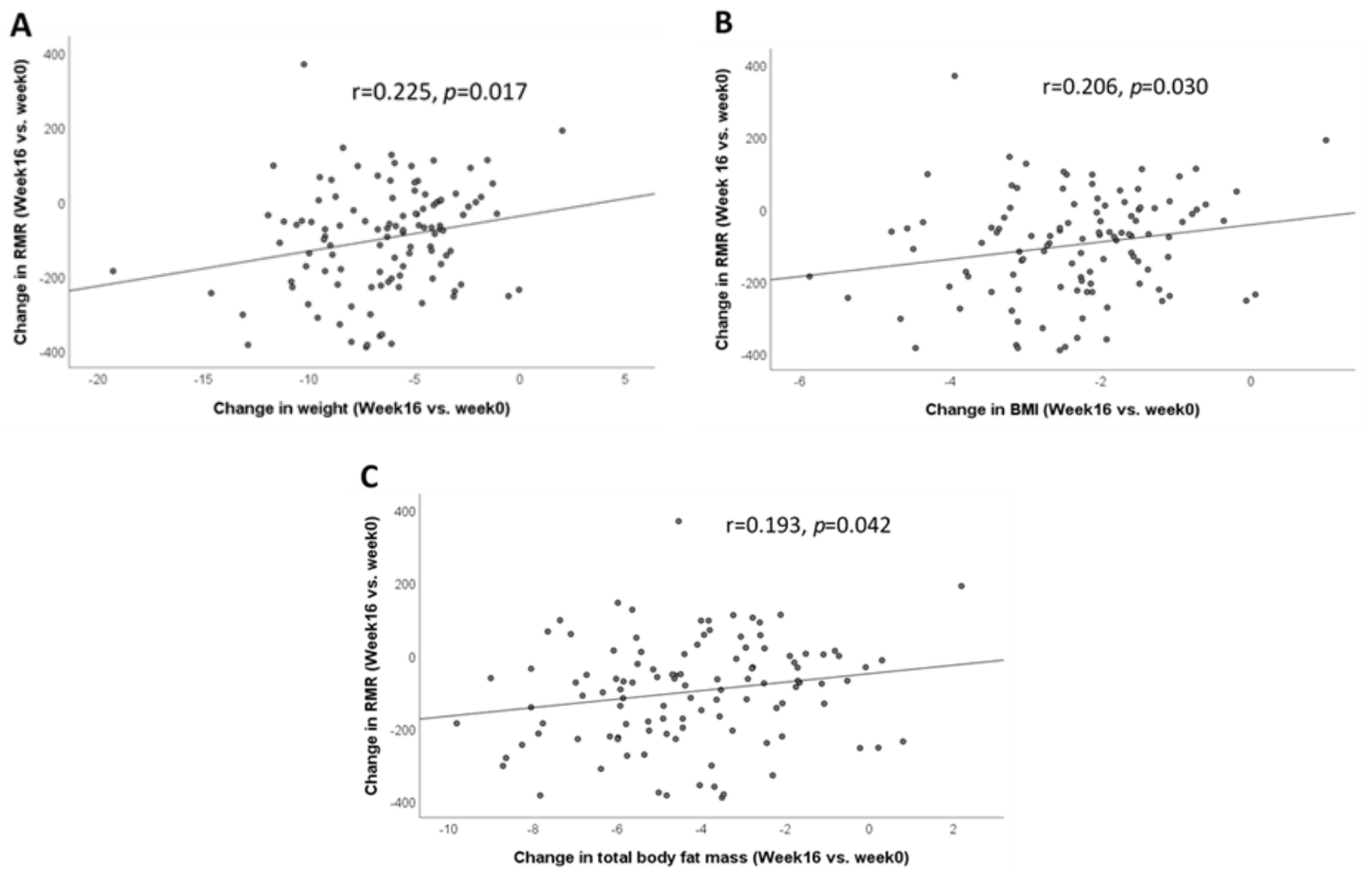

Figure 1. Correlation between change in RMR and change in anthropometric measures after 16 weeks of dietary weight loss intervention (week 16 vs. week 0). (A) Correlation between the change in RMR and the change in weight; (B) correlation between the change in RMR and the change in BMI; (C) correlation between the change in RMR and the change in total body fat mass. Data were analyzed by Spearman's correlation. Abbreviations: body mass index, BMI; resting metabolic rate, $\mathrm{RMR}$.

3.3. Correlation between Change in $R Q$ and Change in Metabolic Measures after 16 Weeks of Weight Loss Intervention (Week 16 vs. Week 0)

The change in RQ was correlated with the change in HDL cholesterol $(r=0.219$, $p=0.021)$, fasting insulin $(\mathrm{r}=0.224, p=0.018)$, HOMA-IR $(\mathrm{r}=0.226, p=0.017)$, and systolic blood pressure $(r=0.217, p=0.022)$ (Figure $2 A-D)$. Within groups, the change in RQ was not correlated with the change in metabolic parameters in the CT and BCAA groups (data not shown). However, the change in RQ was significantly correlated with the change in HDL cholesterol $(r=0.355, p=0.037)$ and percent body fat $(r=-0.329, p=0.041)$ in the HP group. 

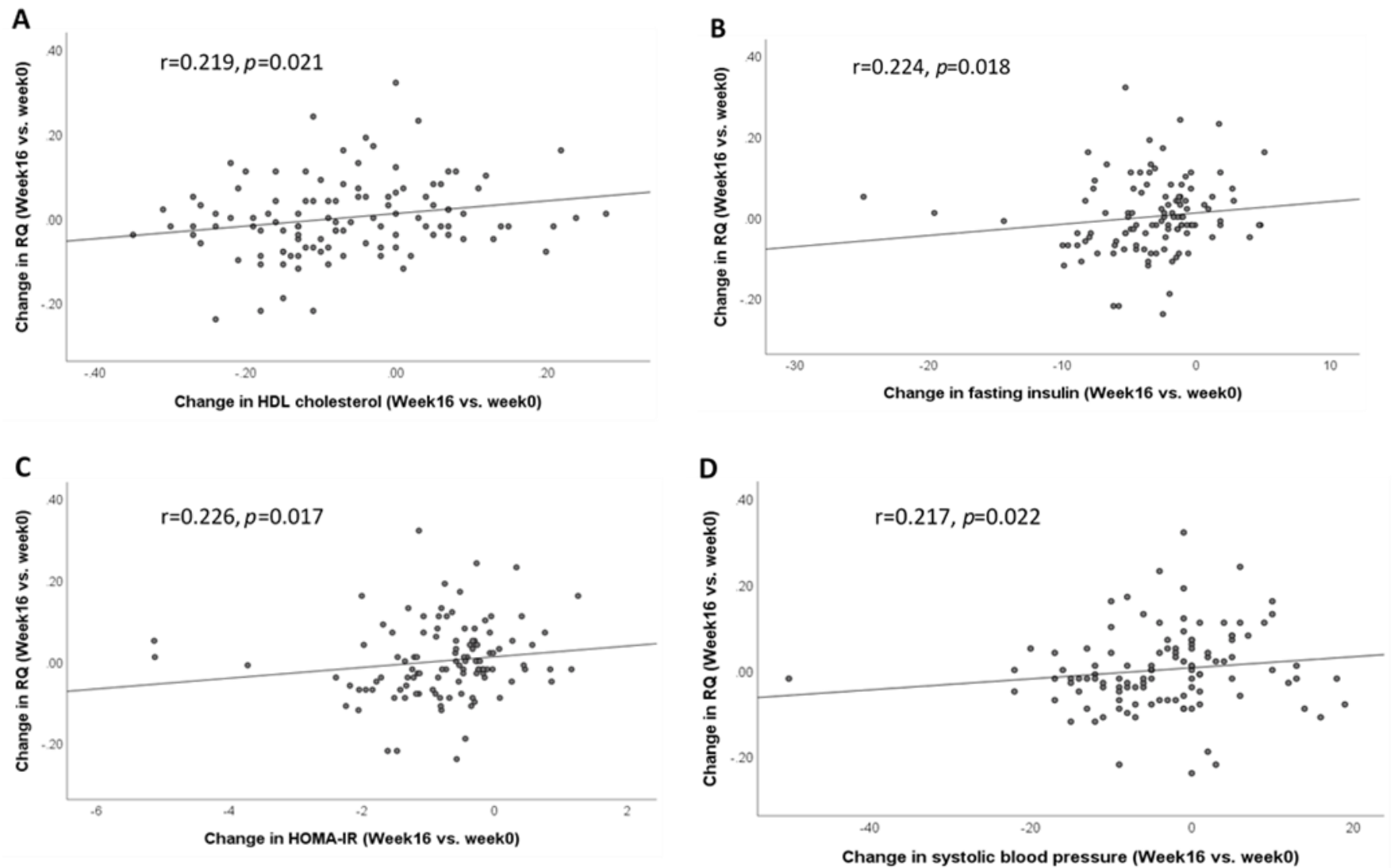

Figure 2. Correlation between the change in RQ and the change in metabolic measures after 16 weeks of dietary weight loss intervention (week 16 vs. week 0). (A) Correlation between the change in RQ and the change in HDL cholesterol; (B) correlation between the change in RQ and the change in fasting insulin; $(C)$ correlation between the change in $R Q$ and the change in HOMA-IR; (D) correlation between the change in RQ and the change in systolic blood pressure. Data were analyzed by Spearman's correlation. Abbreviations: high-density lipoprotein, HDL; homeostatic model assessment of insulin resistance, HOMA-IR; respiratory quotient, RQ.

3.4. Postprandial RQ, Fat Oxidation, Carbohydrate Oxidation, and DIT Responses before and after 16 Weeks of Weight Loss Intervention

Overall, there were no significant differences in mean postprandial responses for fat oxidation, carbohydrate oxidation, and RQ before and after weight loss intervention (Figure 3A-C), except that the mean postprandial DIT response was significantly lower $(p<0.0005)$ after weight loss intervention (Figure 3D). 

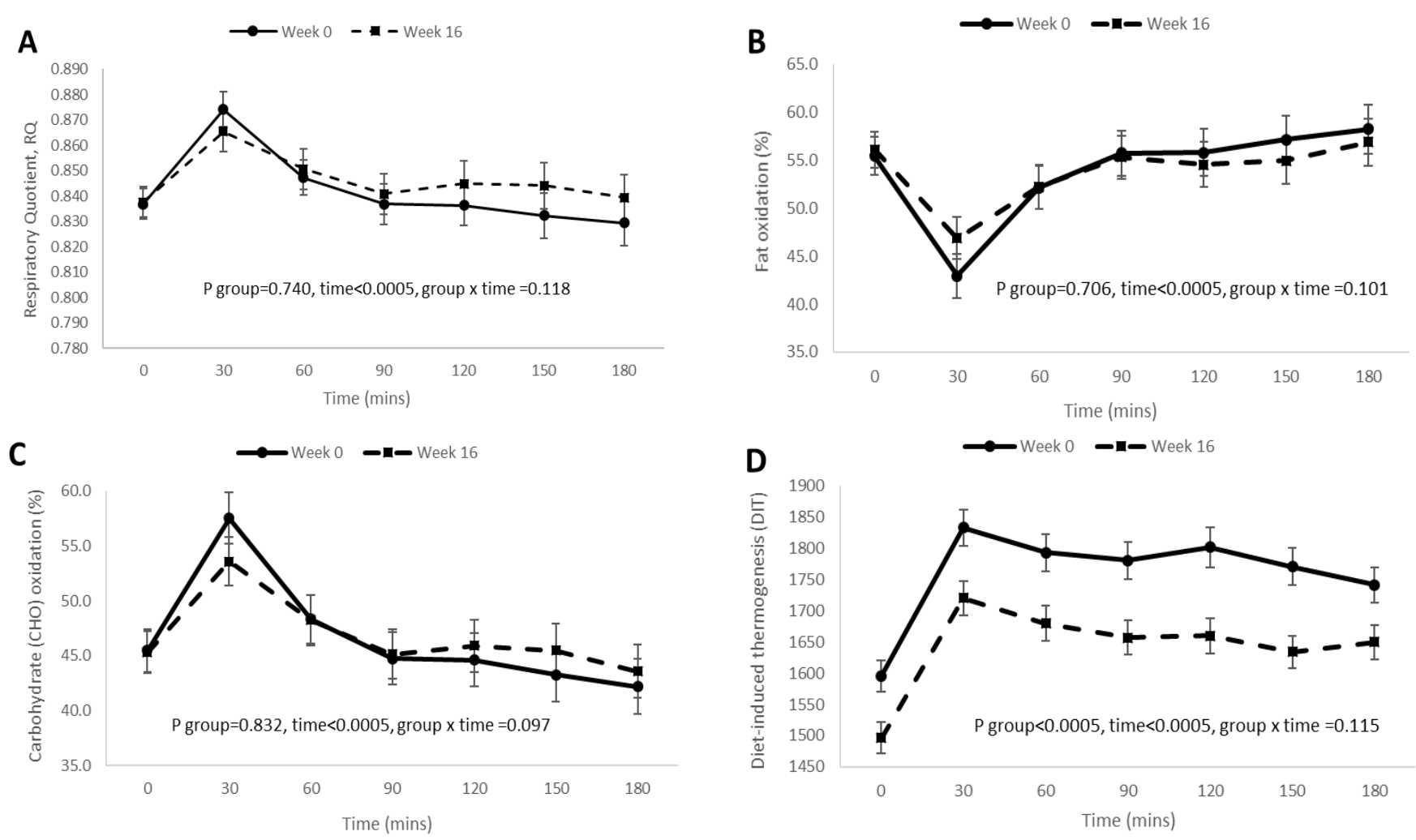

Figure 3. Postprandial RQ, fat oxidation, carbohydrate oxidation, and DIT responses after 16 weeks of weight loss intervention. (A) Postprandial RQ responses after 16 weeks of weight loss intervention; (B) postprandial fat oxidation responses after 16 weeks of weight loss intervention; $(C)$ postprandial carbohydrate oxidation responses after 16 weeks of weight loss intervention; (D) postprandial DIT responses after 16 weeks of weight loss intervention. Data were presented as mean \pm standard error at each time-point and analyzed by repeated measures ANOVA (within subject group).

\subsection{Postprandial RQ, Fat Oxidation, Carbohydrate Oxidation, and DIT Responses among the} Three Diet Groups at Week 16 of Diet Intervention

At baseline (week 0), there were no significant differences in mean postprandial responses for $\mathrm{RQ}$, fat oxidation, carbohydrate oxidation, and DIT among the three diet groups (Figure $\mathrm{S} 2$ ).

After 16 weeks of weight loss intervention, the BCAA diet group had significantly lower mean postprandial RQ $(p=0.028)$ (Figure $4 \mathrm{~A})$ and carbohydrate oxidation $(p=0.044)$ (Figure 4 C), but higher mean postprandial fat oxidation response $(p=0.021)$ (Figure $4 \mathrm{~B}$ ) as compared to the CT group. There were no significant differences in mean postprandial DIT response among the three diet groups $(p=0.232)$ (Figure $4 \mathrm{D})$. The postprandial trajectories for RQ, fat oxidation, carbohydrate oxidation, and DIT were significantly different between the three diet groups (all group $\times$ time interaction $p<0.05$ ) (Figure 4 ). 

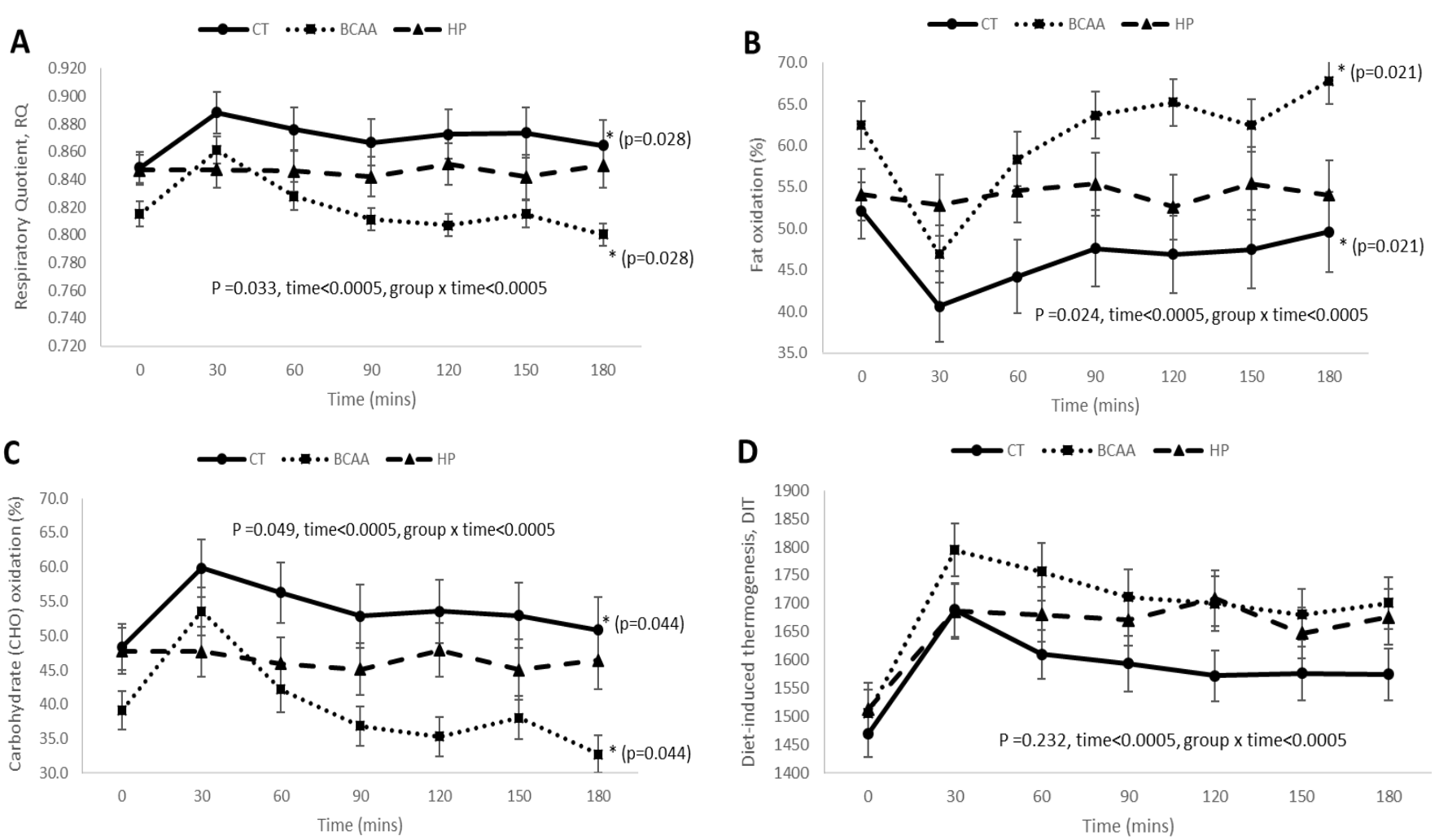

Figure 4. Postprandial RQ, fat oxidation, carbohydrate oxidation, and DIT responses among the three diet groups after 16 weeks of weight loss intervention. (A) Postprandial RQ responses among the 3 diet groups; (B) postprandial fat oxidation responses among the 3 diet groups; (C) postprandial carbohydrate oxidation responses among the 3 diet groups; (D) postprandial DIT responses among the 3 diet groups. Data were presented as mean \pm standard error and analyzed by repeated measures ANOVA (between subject groups). Abbreviations: standard-protein with BCAA, BCAA; standardprotein with placebo, CT; high-protein with placebo, HP. * denotes significant difference of $p<0.05$ between groups.

\section{Discussion}

In this randomized single-blinded, placebo-controlled trial, we showed that there were significant overall improvements in anthropometric measurements and metabolic parameters after 16 weeks of hypocaloric diet intervention [34]. We also observed a significant overall reduction in RMR after 16 weeks of weight loss intervention. Our findings are consistent with other studies that demonstrated a decrease in RMR after weight loss $[8,9]$. However, increasing protein content or supplementing BCAA in the diet did not result in differential effects on anthropometric measurements, metabolic parameters, or RMR.

While human and animal studies have demonstrated that BCAA supplementation could increase RMR [35-37], our data showed that BCAA supplementation was unable to minimize the reduction of RMR after weight loss. The significant decrease in RMR and DIT after weight loss is likely attributed to metabolic adaptation, where the body alters the rate of energy metabolism as a response to weight loss or energy restriction [10,11]. Although lean body mass was reported to be correlated with RMR [5,7], we did not observe a significant correlation between the change in RMR and the change in total body lean mass after weight loss. The decline in RMR is likely due to metabolic adaptation to prevent continuous weight loss and not accounted for by the loss in lean mass [49]. RQ reflects substrate utilization and the change in RQ represents a change in the type of substrate, i.e., carbohydrate and fat that is oxidized for energy metabolism [48]. The utilization of different substrates has been implicated in metabolic complications [50], and we showed that the change in $R Q$ (substrate utilization) was positively correlated with the change 
in metabolic outcomes such as HDL cholesterol, fasting insulin, HOMA-IR, and systolic blood pressure.

Our data also demonstrated a significant difference in the postprandial $R Q$, fat oxidation, and carbohydrate oxidation responses between hypocaloric standard-protein diet with and without BCAA supplementation (BCAA vs. CT). BCAA supplementation significantly lowered the postprandial $R Q$, indicating a higher fat but lower carbohydrate oxidation response, suggesting that BCAA-supplemented weight loss intervention might promote postprandial fat oxidation response. Further studies are required to examine the long-term effect of the BCAA-induced postprandial fat oxidation response on body fat composition. BCAA supplementation has been found to increase lipid oxidation during exercise in glycogen-depleted subjects [39]. Mourier et al. showed that a BCAA-supplemented hypocaloric diet produced the highest body fat loss in male wrestlers with a high level of exercise performance as compared to hypocaloric diets without BCAA supplementation, and it was postulated that the fat loss may be caused by specific hormonal adaptations induced by BCAA [32]. Dudgeon et al. also showed that consumption of a BCAA-supplemented hypocaloric diet during resistance training caused a greater decrease in body fat percentage as compared to hypocaloric diet without BCAA supplementation [31]. The differences in study cohort, caloric value of diet, dosage of BCAA supplements, duration of study, and exercise intensity between our study and those of Mourier et al. and Dudgeon et al. [31,32] may explain the absence of significant body fat loss between BCAA and CT groups in our study. The decreased rate of carbohydrate oxidation within the BCAA group after 16 weeks of weight loss intervention may indicate the preferential ability of BCAA-supplemented diet in oxidizing fat instead of carbohydrate. L-Leucine activates mTOR signaling pathway to stimulate protein synthesis in the skeletal muscle and promote mitochondrial biogenesis, which results in increased cellular respiration. The increase in cellular energy metabolism stimulates fatty acid oxidation [51]. Hence, BCAA supplementation to a diet that is low in carbohydrate and high in fat may promote greater body fat loss [52].

The strength of our study lies in it being a randomized controlled trial, which included the supply of meals and monitoring of food intake, and BCAA supplementation was provided for a longer period of 16 weeks to a larger cohort of subjects $(n=35)$ as compared to previous studies [53]. The main limitation in this study is that we were unable to calculate protein oxidation as we did not measure urinary nitrogen excretion. However, the aim of our study was to examine the effect of diets of different protein content on RMR and substrate utilization in terms of $R Q$, and thus the information on protein oxidation would not add to the results of this study.

\section{Conclusions}

In conclusion, BCAA supplementation to a standard-protein hypocaloric diet did not significantly minimize the decrease in RMR compared to standard-protein and highprotein hypocaloric diets without BCAA supplementation. However, the postprandial fat oxidation response was higher after BCAA-supplemented weight loss intervention.

Supplementary Materials: The following are available online at https:/ / www.mdpi.com/article/10 .3390/nu13124245/s1, Table S1: Baseline characteristics of study participants in the three diet groups. Table S2: Clinical characteristics of study participants before (week 0) and after (week 16) dietary weight loss intervention. Figure S1: Consort diagram for recruitment, screening, enrolment, and randomization of study subjects. Figure S2: Postprandial RQ, fat oxidation, carbohydrate oxidation, and DIT responses among the three diet groups at baseline (week 0 ).

Author Contributions: Conceptualization, D.S.Q.O., M.F.F.C., C.M.K. and Y.S.L.; Methodology, M.F.F.C., C.M.K. and Y.S.L.; Formal Analysis, D.S.Q.O., C.M.K. and Y.S.L.; Investigation, E.S.T., C.J.H., M.K.S.L., E.Y.H.K., C.S.T., M.F.F.C., C.M.K. and Y.S.L.; Resources, C.J.H., M.F.F.C., C.M.K. and Y.S.L.; Data Curation, D.S.Q.O., J.Q.R.L., F.Y.O., M.F.F.C., C.M.K. and Y.S.L.; Writing-Original Draft Preparation, D.S.Q.O., C.M.K. and Y.S.L.; Writing-Review \& Editing, D.S.Q.O., J.Q.R.L., F.Y.O., M.F.F.C., C.M.K., Y.S.L., E.S.T., C.J.H., M.K.S.L., E.Y.H.K. and C.S.T.; Visualization, D.S.Q.O., C.M.K. and Y.S.L.; Supervision, M.F.F.C., C.M.K. and Y.S.L.; Project Administration, D.S.Q.O., J.Q.R.L. and 
F.Y.O.; Funding Acquisition, M.F.F.C. and Y.S.L. All authors have read and agreed to the published version of the manuscript.

Funding: This research was funded by the Clinical Scientist-Individual Research Grant administered by Ministry of Health's National Medical Research Council (NMRC), Singapore-NMRC/CIRG/1375/2013. Additional funding is provided by the Singapore Institute for Clinical Sciences, Agency for Science Technology and Research (A*STAR), Singapore.

Institutional Review Board Statement: The study was conducted according to the guidelines of the Declaration of Helsinki and approved by the Domain Specific Review Board of National Healthcare Group, Singapore (protocol code: 2014/00085 and date of approval: 21 March 2014).

Informed Consent Statement: Informed consent was obtained from all subjects involved in the study.

Data Availability Statement: The data presented in this study are available within the article or supplementary material.

Acknowledgments: We would like to acknowledge the kind help, assistance, and support provided by Ruth Sasha Siva, Ng Yuen Fann, Yap Siew Moi, Lim Ying Yan, Janelle Limqueco, Michele Wong, Christine Ng, Yu Shao Mei, and Wu Ting.

Conflicts of Interest: The authors declare no conflict of interest. The funders had no role in the design of the study; in the collection, analyses, or interpretation of data; in the writing of the manuscript; or in the decision to publish the results.

\section{References}

1. Ravussin, E.; Gautier, J. Metabolic predictors of weight gain. Int. J. Obes. 1999, 23, S37-S41. [CrossRef]

2. Speakman, J.R.; Selman, C. Physical activity and resting metabolic rate. Proc. Nutr. Soc. 2003, 62, 621-634. [CrossRef]

3. Westerterp, K.R. Control of energy expenditure in humans. Eur. J. Clin. Nutr. 2017, 71, 340-344. [CrossRef]

4. Cunningham, J.J. Body composition as a determinant of energy expenditure: A synthetic review and a proposed general prediction equation. Am. J. Clin. Nutr. 1991, 54, 963-969. [CrossRef] [PubMed]

5. Nelson, K.M.; Weinsier, R.L.; Long, C.L.; Schutz, Y. Prediction of resting energy expenditure from fat-free mass and fat mass. Am. J. Clin. Nutr. 1992, 56, 848-856. [CrossRef] [PubMed]

6. Sparti, A.; DeLany, J.; de la Bretonne, J.A.; Sander, G.E.; Bray, G.A. Relationship between resting metabolic rate and the composition of the fat-free mass. Metabolism 1997, 46, 1225-1230. [CrossRef]

7. Ravussin, E.; Burnand, B.; Schutz, Y.; Jéquier, E. Twenty-four-hour energy expenditure and resting metabolic rate in obese, moderately obese, and control subjects. Am. J. Clin. Nutr. 1982, 35, 566-573. [CrossRef] [PubMed]

8. Weinsier, R.L.; Hunter, G.R.; Zuckerman, P.A.; Redden, D.T.; Darnell, B.E.; Larson, D.E.; Newcomer, B.R.; Goran, M.I. Energy expenditure and free-living physical activity in black and white women: Comparison before and after weight loss. Am. J. Clin. Nutr. 2000, 71, 1138-1146. [CrossRef]

9. Goele, K.; Bosy-Westphal, A.; Rümcker, B.; Lagerpusch, M.; Müller, M.J. Influence of changes in body composition and adaptive thermogenesis on the difference between measured and predicted weight loss in obese women. Obes. Facts 2009, 2, 105-109. [CrossRef]

10. Tremblay, A.; Royer, M.-M.; Chaput, J.-P.; Doucet, E. Adaptive thermogenesis can make a difference in the ability of obese individuals to lose body weight. Int. J. Obes. 2013, 37, 759-764. [CrossRef]

11. Doucet, E.; St-Pierre, S.; Alméras, N.; Després, J.-P.; Bouchard, C.; Tremblay, A. Evidence for the existence of adaptive thermogenesis during weight loss. Br. J. Nutr. 2001, 85, 715-723. [CrossRef] [PubMed]

12. Müller, M.J.; Enderle, J.; Bosy-Westphal, A. Changes in Energy Expenditure with Weight Gain and Weight Loss in Humans. Curr. Obes. Rep. 2016, 5, 413-423. [CrossRef] [PubMed]

13. Trexler, E.T.; Smith-Ryan, A.E.; Norton, L.E. Metabolic adaptation to weight loss: Implications for the athlete. J. Int. Soc. Sports Nutr. 2014, 11, 7. [CrossRef] [PubMed]

14. Ravussin, E.; Lillioja, S.; Knowler, W.C.; Christin, L.; Freymond, D.; Abbott, W.G.; Boyce, V.; Howard, B.V.; Bogardus, C. Reduced Rate of Energy Expenditure as a Risk Factor for Body-Weight Gain. N. Engl. J. Med. 1988, 318, 467-472. [CrossRef] [PubMed]

15. Buscemi, S.; Verga, S.; Caimi, G.; Cerasola, G. Low relative resting metabolic rate and body weight gain in adult Caucasian Italians. Int. J. Obes. 2005, 29, 287-291. [CrossRef]

16. Rosenbaum, M.; Leibel, R.L. Adaptive thermogenesis in humans. Int. J. Obes. 2010, 34, S47-S55. [CrossRef] [PubMed]

17. Weyer, C.; Pratley, R.E.; Salbe, A.D.; Bogardus, C.; Ravussin, E.; Tataranni, P.A. Energy Expenditure, Fat Oxidation, and Body Weight Regulation: A Study of Metabolic Adaptation to Long- Term Weight Change. J. Clin. Endocrinol. Metab. 2000, 85, 1087-1094. [CrossRef]

18. Shook, R.P.; Hand, G.A.; Paluch, A.E.; Wang, X.; Moran, R.G.; Hebert, J.R.; Jakicic, J.M.; Blair, S.N. High respiratory quotient is associated with increases in body weight and fat mass in young adults. Eur. J. Clin. Nutr. 2016, 70, 1197-1202. [CrossRef] 
19. Hirsch, K.R.; Smith-Ryan, A.E.; Blue, M.N.; Mock, M.G.; Trexler, E.T. Influence of segmental body composition and adiposity hormones on resting metabolic rate and substrate utilization in overweight and obese adults. J. Endocrinol. Investig. 2017, 40, 635-643. [CrossRef] [PubMed]

20. Lightowler, H.; Schweitzer, L.; Theis, S.; Henry, C.J. Changes in Weight and Substrate Oxidation in Overweight Adults Following Isomaltulose Intake During a 12-Week Weight Loss Intervention: A Randomized, Double-Blind, Controlled Trial. Nutrients 2019, 11, 2367. [CrossRef]

21. Carbone, J.W.; Pasiakos, S.M. Dietary Protein and Muscle Mass: Translating Science to Application and Health Benefit. Nutrients 2019, 11, 1136. [CrossRef]

22. Sahni, S.; Mangano, K.; Hannan, M.T.; Kiel, D.; McLean, R.R. Higher Protein Intake Is Associated with Higher Lean Mass and Quadriceps Muscle Strength in Adult Men and Women. J. Nutr. 2015, 145, 1569-1575. [CrossRef] [PubMed]

23. Farnsworth, E.; Luscombe, N.D.; Noakes, M.; Wittert, G.; Argyiou, E.; Clifton, P.M. Effect of a high-protein, energy-restricted diet on body composition, glycemic control, and lipid concentrations in overweight and obese hyperinsulinemic men and women. Am. J. Clin. Nutr. 2003, 78, 31-39. [CrossRef]

24. Verreijen, A.M.; Verlaan, S.; Engberink, M.F.; Swinkels, S.; Bosch, J.D.V.-V.D.; Weijs, P.J.M. A high whey protein-, leucine-, and vitamin D-enriched supplement preserves muscle mass during intentional weight loss in obese older adults: A double-blind randomized controlled trial. Am. J. Clin. Nutr. 2015, 101, 279-286. [CrossRef]

25. Longland, T.M.; Oikawa, S.Y.; Mitchell, C.J.; Devries, M.C.; Phillips, S.M. Higher compared with lower dietary protein during an energy deficit combined with intense exercise promotes greater lean mass gain and fat mass loss: A randomized trial. Am. J. Clin. Nutr. 2016, 103, 738-746. [CrossRef] [PubMed]

26. Arciero, P.J.; Edmonds, R.; He, F.; Ward, E.; Gumpricht, E.; Mohr, A.; Ormsbee, M.J.; Astrup, A. Protein-Pacing Caloric-Restriction Enhances Body Composition Similarly in Obese Men and Women during Weight Loss and Sustains Efficacy during Long-Term Weight Maintenance. Nutrients 2016, 8, 476. [CrossRef]

27. Schiavo, L.; Scalera, G.; Pilone, V.; de Sena, G.; Quagliariello, V.; Iannelli, A.; Barbarisi, A. A Comparative Study Examining the Impact of a Protein-Enriched Vs Normal Protein Postoperative Diet on Body Composition and Resting Metabolic Rate in Obese Patients after Sleeve Gastrectomy. Obes. Surg. 2017, 27, 881-888. [CrossRef]

28. Spillane, M.; Schwarz, N.; Willoughby, D.S. Heavy resistance training and peri-exercise ingestion of a multi-ingredient ergogenic nutritional supplement in males: Effects on body composition, muscle performance and markers of muscle protein synthesis. $J$. Sports Sci. Med. 2014, 13, 894-903.

29. AbuMoh'd, M.F.; Matalqah, L.; Al-Abdulla, Z. Effects of Oral Branched-Chain Amino Acids (BCAAs) Intake on Muscular and Central Fatigue During an Incremental Exercise. J. Hum. Kinet. 2020, 72, 69-78. [CrossRef] [PubMed]

30. Shimomura, Y.; Inaguma, A.; Watanabe, S.; Yamamoto, Y.; Muramatsu, Y.; Bajotto, G.; Sato, J.; Shimomura, N.; Kobayashi, H.; Mawatari, K.; et al. Branched-Chain Amino Acid Supplementation Before Squat Exercise and Delayed-Onset Muscle Soreness. Int. J. Sport Nutr. Exerc. Metab. 2010, 20, 236-244. [CrossRef]

31. Dudgeon, W.D.; Kelley, E.P.; Scheett, T.P. In a single-blind, matched group design: Branched-chain amino acid supplementation and resistance training maintains lean body mass during a caloric restricted diet. J. Int. Soc. Sports Nutr. 2016, 13, 1-10. [CrossRef]

32. Mourier, A.; Bigard, A.X.; de Kerviler, E.; Roger, B.; Legrand, H.; Guezennec, C.Y. Combined Effects of Caloric Restriction and Branched-Chain Amino Acid Supplementation on Body Composition and Exercise Performance in Elite Wrestlers. Int. J. Sports Med. 1997, 18, 47-55. [CrossRef]

33. Novin, Z.S.; Ghavamzadeh, S.; Mehdizadeh, A. The Weight Loss Effects of Branched Chain Amino Acids and Vitamin B6: A Randomized Controlled Trial on Obese and Overweight Women. Int. J. Vitam. Nutr. Res. 2018, 88, 80-89. [CrossRef]

34. Ooi, D.S.Q.; Ling, J.Q.R.; Sadananthan, S.A.; Velan, S.S.; Ong, F.Y.; Khoo, C.M.; Tai, E.S.; Henry, C.J.; Leow, M.K.S.; Khoo, E.Y.H.; et al. Branched-Chain Amino Acid Supplementation Does Not Preserve Lean Mass or Affect Metabolic Profile in Adults with Overweight or Obesity in a Randomized Controlled Weight Loss Intervention. J. Nutr. 2021, 151, 911-920. [CrossRef] [PubMed]

35. Roelofs, E.J.; Smith-Ryan, A.E. Effects of Branched-chain Amino Acids on Resting Metabolic Rate, Body Composition, And Satiety in Females. Med. Sci. Sports Exerc. 2017, 49, 81. [CrossRef]

36. Tamburo, S.; Sponsiello, N. Short-term effects of BCAA, Arginine and CLA supplementation on rest energy expenditure and body composition. In Proceedings of the 16th Annual Congress of the European College of Sport Science-Ecss, Liverpool, UK, 6-9 July 2011; Volume 18, pp. 47-55.

37. She, P.; Reid, T.M.; Bronson, S.; Vary, T.C.; Hajnal, A.; Lynch, C.J.; Hutson, S.M. Disruption of BCATm in Mice Leads to Increased Energy Expenditure Associated with the Activation of a Futile Protein Turnover Cycle. Cell Metab. 2007, 6, 181-194. [CrossRef] [PubMed]

38. McGarrah, R.W.; Zhang, G.-F.; Christopher, B.A.; Deleye, Y.; Walejko, J.M.; Page, S.; Ilkayeva, O.; White, P.J.; Newgard, C.B. Dietary branched-chain amino acid restriction alters fuel selection and reduces triglyceride stores in hearts of Zucker fatty rats. Am. J. Physiol. Metab. 2020, 318, E216-E223. [CrossRef] [PubMed]

39. Gualano, B.; Bozza, T.; Campos-Ferraz, P.; Roschel, H.; Costa, A.; Marquezi, M.; Benatti, F.; Lancha, A., Jr. Branched chain amino acids supplementation enhance exercise capacity and lipid oxidation during endurance exercise after muscle glycogen depletion. J. Sports Med. Phys. Fit. 2011, 51, 82-88.

40. Faul, F.; Erdfelder, E.; Lang, A.-G.; Buchner, A. G*Power 3: A flexible statistical power analysis program for the social, behavioral, and biomedical sciences. Behav. Res. Methods 2007, 39, 175-191. [CrossRef] 
41. Fouré, A.; Nosaka, K.; Gastaldi, M.; Mattei, J.-P.; Boudinet, H.; Guye, M.; Vilmen, C.; Le Fur, Y.; Bendahan, D.; Gondin, J. Effects of branched-chain amino acids supplementation on both plasma amino acids concentration and muscle energetics changes resulting from muscle damage: A randomized placebo-controlled trial. Clin. Nutr. 2016, 35, 83-94. [CrossRef]

42. Shimomura, Y.; Kobayashi, H.; Mawatari, K.; Akita, K.; Inaguma, A.; Watanabe, S.; Bajotto, G.; Sato, J. Effects of squat exercise and branched-chain amino acid supplementation on plasma free amino acid concentrations in young women. J. Nutr. Sci. Vitaminol. 2009, 55, 288-291. [CrossRef] [PubMed]

43. Bifari, F.; Nisoli, E. Branched-chain amino acids differently modulate catabolic and anabolic states in mammals: A pharmacological point of view. Br. J. Pharmacol. 2017, 174, 1366-1377. [CrossRef] [PubMed]

44. Wallace, T.M.; Levy, J.C.; Matthews, D.R. Use and Abuse of HOMA Modeling. Diabetes Care 2004, 27, 1487-1495. [CrossRef]

45. Luscombe, N.D.; Tsopelas, C.; Bellon, M.; Clifton, P.M.; Kirkwood, I.; Wittert, G.A. Use of [14C]-sodium bicarbonate/urea to measure total energy expenditure in overweight men and women before and after low calorie diet induced weight loss. Asia Pac. J. Clin. Nutr. 2006, 15, 307-316.

46. Henry, J.; Lightowler, H.J.; Marchini, J. Intra-individual variation in resting metabolic rate during the menstrual cycle. Br. J. Nutr. 2003, 89, 811-817. [CrossRef]

47. Weir, J.B. New methods for calculating metabolic rate with special reference to protein metabolism. 1949. Nutrition 1990, 6 , 213-221.

48. Zurlo, F.; Lillioja, S.; Puente, A.E.-D.; Nyomba, B.L.G.; Raz, I.; Saad, M.F.; Swinburn, B.A.; Knowler, W.C.; Bogardus, C.; Ravussin, E. Low ratio of fat to carbohydrate oxidation as predictor of weight gain: Study of 24-h RQ. Am. J. Physiol. Metab. 1990, 259, E650-E657. [CrossRef] [PubMed]

49. Heshka, S.; Yang, M.U.; Wang, J.; Burt, P.; Pi-Sunyer, F.X. Weight loss and change in resting metabolic rate. Am. J. Clin. Nutr. 1990, 52, 981-986. [CrossRef]

50. Pujia, A.; Mazza, E.; Ferro, Y.; Gazzaruso, C.; Coppola, A.; Doldo, P.; Grembiale, R.D.; Pujia, R.; Romeo, S.; Montalcini, T. Lipid Oxidation Assessed by Indirect Calorimetry Predicts Metabolic Syndrome and Type 2 Diabetes. Front. Endocrinol. 2018, 9. [CrossRef]

51. Duan, Y.; Li, F.; Liu, H.; Li, Y.; Liu, Y.; Kong, X.; Zhang, Y.; Deng, D.; Tang, Y.; Feng, Z.; et al. Nutritional and regulatory roles of leucine in muscle growth and fat reduction. Front. Biosci. 2015, 20, 796-813. [CrossRef]

52. Evangeliou, A.; Spilioti, M.; Doulioglou, V.; Kalaidopoulou, P.; Ilias, A.; Skarpalezou, A.; Katsanika, I.; Kalamitsou, S.; Vasilaki, K.; Chatziioanidis, I.; et al. Branched Chain Amino Acids as Adjunctive Therapy to Ketogenic Diet in Epilepsy: Pilot Study and Hypothesis. J. Child Neurol. 2009, 24, 1268-1272. [CrossRef] [PubMed]

53. Fouré, A.; Bendahan, D. Is Branched-Chain Amino Acids Supplementation an Efficient Nutritional Strategy to Alleviate Skeletal Muscle Damage? A Systematic Review. Nutrients 2017, 9, 1047. [CrossRef] [PubMed] 